Polymer Journal, Vol. 39, No. 6, pp. 537-542 (2007)

(C) 2007 The Society of Polymer Science, Japan

\title{
Crystallization and Morphology Development of Binary Crystalline Poly(ethylene succinate)/Poly(ethylene oxide) (PES/PEO) Blend with Non-Isothermal Crystallization
}

\author{
Shuangai HE and Jieping LIU ${ }^{\dagger}$ \\ Department of Chemistry, Huaibei Coal Industry Teachers College, Anhui, 235000 China
}

(Received October 26, 2006; Accepted March 5, 2007; Published April 17, 2007)

\begin{abstract}
Spherulitic growth and morphology of PES/PEO blend with non-isothermal crystallization were investigated by means of differential scanning calorimetry and polarizing optical microscope. Depending on the crystallization conditions and compositions, PES and PEO can crystallize either simultaneously at the same temperature or separately at different temperatures, i.e., step-crystallization. An opposed-interpenetrated growth was found in the simultaneous crystallization process of PES and PEO. PES and PEO spherulite nucleated with the same nucleus and the growth faces of PES and PEO lamellae grow simultaneously with different growth rate in the same spherulite. The PES lamellae radically developed along the preexisting PEO lamellae, resulting in the change of spherulitic morphology. The change of spherulitic morphology for the step-crystallization process of PES and PEO was also discussed. [doi:10.1295/polymj.PJ2006139]

KEY WORDS PES/PEO Blend / Crystallization and Morphology Development / OpposedInterpenetrated Growth / Non-Isothermal Crystallization /
\end{abstract}

Polymer blends including semicrystalline/amorphous and semicrystalline/semicrystalline blends can exhibit a complicated phase behavior and variety of morphology and structure. The morphological development and spherulitic growth of polymer blends had been a subject of continuing interest for researchers from both scientific and industrial fields. ${ }^{1-5}$ Some new crystallization behaviors and morphologies have been investigated in polymer blends. For example, a unique growth behavior, i.e., interpenetrated growth, has been found in binary crystalline polymer blends. ${ }^{6-10}$ The growth front of spherulites of one component can penetrate into spherulites of other component to grow continually under certain conditions. This phenomenon has received much attention immediately due to its contribution to polymer physics.

It is well known that, depending on the crystallization temperature and composition, the second component (low temperature crystallizing component) may be included in or rejected from the spherulites of the first component (high temperature crystallizing component) for a binary crystalline polymer blend since the second component is still in melting state when the first component crystallize. ${ }^{11-13}$ When the melt phase of the second component is engulfed in the spherulites of the first component, the second component has to crystallize inside the spherulites formed by the first component when the crystallization temperature was decreased to the range of the crystallization temperature of the second component. It was reported that the melt phase may exist in interlamellar or inter- fibrillar regions. ${ }^{14,15}$ Therefore the crystallization of the second component should be controlled not only by the crystallization conditions but also by the spherulitic structure of the first component and the existence region of the melt phase. So far many problems about this crystallization behavior are not yet clear due to its complexity.

Normally, less binary crystalline polymer pair with complete miscibility has been found. Poly(ethylene succinate)/poly(ethylene oxide) (PES/PEO) is a very useful polymer pair for studying the structure and morphology development of binary crystalline polymer blend due to their miscibility and availability. ${ }^{8,15}$ The crystallization behaviors and spherulitic growth of PES/PEO blend have been studied under the isothermal crystallization conditions, and a interpenetrated crystallization behavior has been observed. ${ }^{8}$ In this paper, a unique crystallization behavior with PES and PEO growth from same nucleus in the same spherulite under non-isothermal crystallization conditions was observed. This novel crystallization behavior and morphology development of PEO/PES blends during non-isothermal crystallization processes of the blends was discussed.

\section{EXPERIMENTAL}

PEO $\left(M_{\mathrm{w}}=100000, T_{\mathrm{m}} \sim 67^{\circ} \mathrm{C}\right)$ and PES $\left(M_{\mathrm{w}}=\right.$ $11000, T_{\mathrm{m}} \sim 103^{\circ} \mathrm{C}$ ) used in this study were purchased from Acros Organics. The blended samples were dissolved in the desired ratio in $\mathrm{N}, \mathrm{N}$-dimethyl-

${ }^{\dagger}$ To whom correspondence should be addressed (E-mail: jpliu@ @bcnc.edu.cn). 


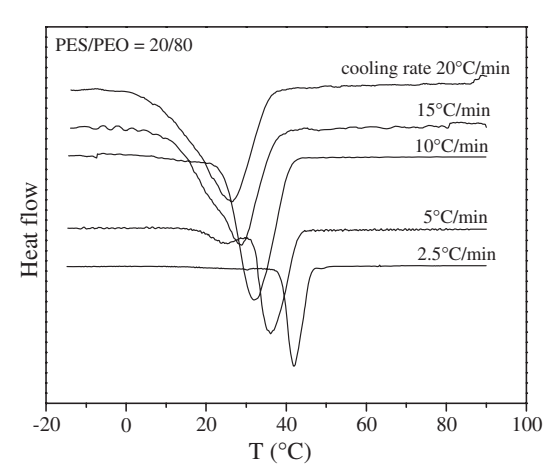

(a)

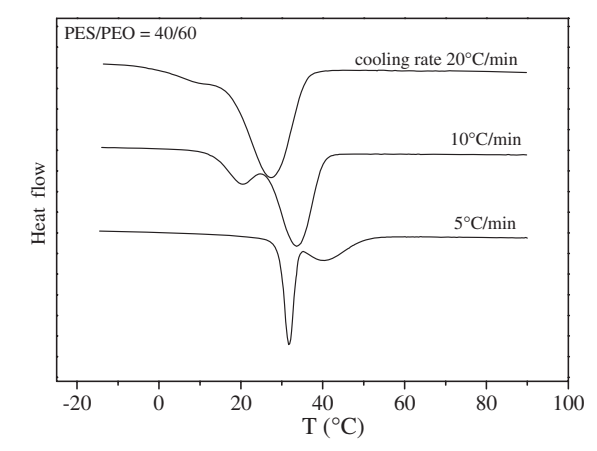

(c)

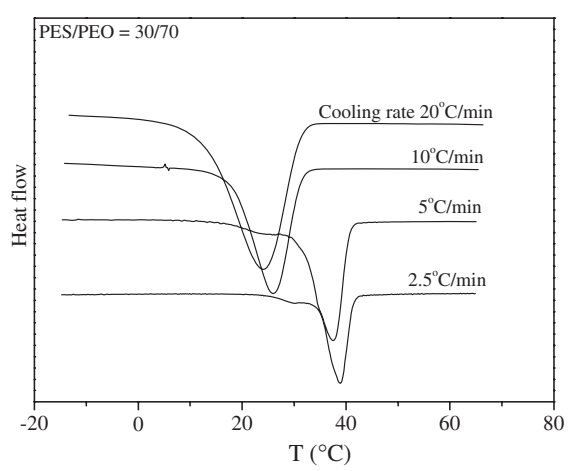

(b)

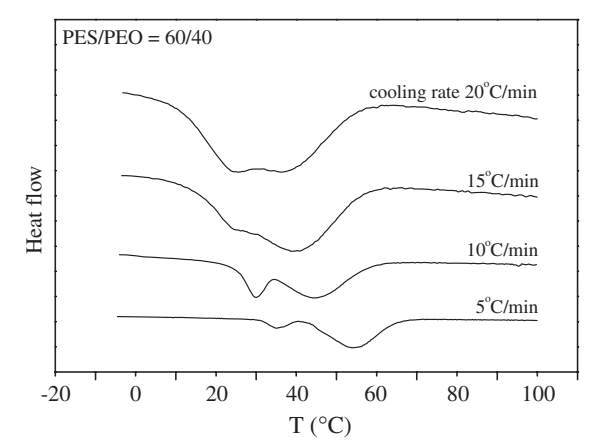

(d)

Figure 1. The DSC curves of PES/PEO blends with different compositions at different cooling rate.

formamide (DMF) to form $10 \%$ dilute solution at about $80^{\circ} \mathrm{C}$. The solvent was evaporated slowly at room temperature in a Petri dish to form a homogeneous film, which was further dried in vacuum for two weeks.

Non-isothermal crystallization experiments were carried out by differential scanning calorimetry (DSC, Netzsch DSC204-F1). The samples sealed in aluminum pans were first heated up to $130^{\circ} \mathrm{C}$ for $5 \mathrm{~min}$ to get a equilibrium melt and then cooled down to $-20^{\circ} \mathrm{C}$ with different cooling rate. The DSC curves were recorded to analyze the crystallization processes of PEO and PES.

Spherulitic morphology was observed with a polarizing optical microscope (Nikon Eclipse E400) and a microscopy heat stage (Instec STC200). The thin film sample with thickness of $10-20 \mu \mathrm{m}$ sandwiched between two optical glass slides was put on the microscopy heat stage. The sample was first heated to $130^{\circ} \mathrm{C}$ in atmospheric ambience for $10 \mathrm{~min}$ to get a equilibrium melt and then cooled down to the room temperature at different cooling rate. The development of spherulitic morphology at different temperature was observed.

\section{RESULTS AND DISCUSSION}

Figure 1 showed the DSC curves of PES/PEO blends with different compositions and cooling rates.
It is found that the crystallization temperatures of PEO and PES are dependent on the composition of blends and cooling rate. PEO and PES can crystallize either simultaneously at the same crystallization temperature or separately at different temperatures. When PES content is more than $40 \%$ (wt) two crystallization peaks in DSC curves can be observed in the range of cooling rate used here, indicating that PES and PEO separately crystallize at different temperatures, i.e., step-crystallization. For 20/80 (PES/PEO) and 30/ 70 (PES/PEO) compositions, only one crystallization peak was observed in DSC curves at cooling rate of $20^{\circ} \mathrm{C}$ and $10^{\circ} \mathrm{C}$, showing that PEO and PES crystallize simultaneously in these cases. However, it is difficult to analyze crystallization behaviors of PEO and PES from the DSC curves. The crystallization behaviors and morphology development of PEO and PES were then further investigated by using polarizing optical microcopy.

Figure 2 showed the non-isothermal crystallization process of PES/PEO blend with $\mathrm{PES} / \mathrm{PEO}=20 / 80$ at cooling rate of $5{ }^{\circ} \mathrm{C}$. A novel spherulitic growth has been observed. It is found that two growth fronts grew simultaneously with the same nucleus in the same spherulite (arrow A and B in Figure $2 \mathrm{~b}$ and $2 c)$. The growth rates of two growth surfaces are different. The final spherulite morphologies formed by these growth surfaces are also different. The open-spherulite (small density of lamellar stacks) 


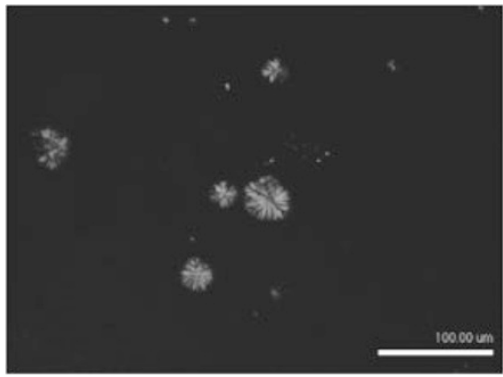

(a) $T=50.4^{\circ} \mathrm{C}$

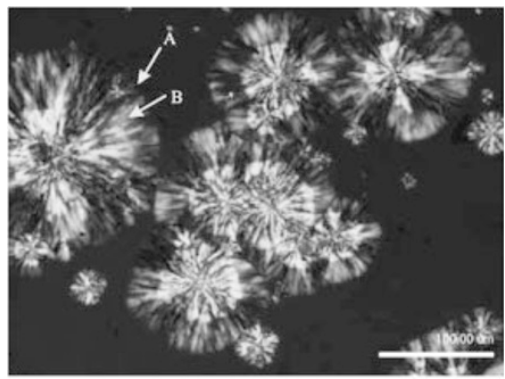

(c) $T=44.3^{\circ} \mathrm{C}$

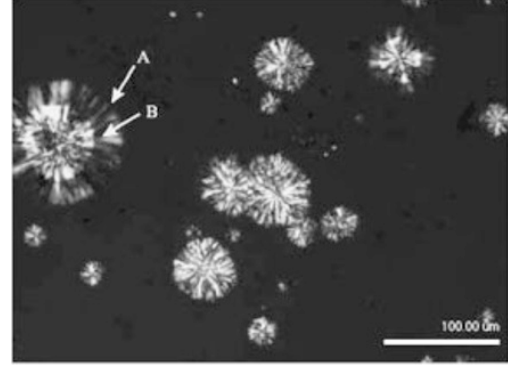

(b) $T=45.8^{\circ} \mathrm{C}$

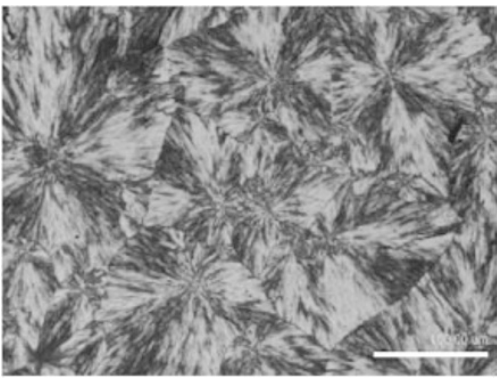

(d) $T=35.7^{\circ} \mathrm{C}$

Figure 2. Spherulitic morphology of PES/PEO $=20 / 80$ blend at cooling rate of $5^{\circ} \mathrm{C}$ investigated by polarizing optical microscope with temperature of (a) $T=50.4^{\circ} \mathrm{C}$, (b) $T=45.8^{\circ} \mathrm{C}$, (c) $T=44.3^{\circ} \mathrm{C}$, (d) $T=35.7^{\circ} \mathrm{C}$ respectively. Two growth fronts can be observed clearly in the same spherulite (arrow A and B in Figure $2 \mathrm{~b}$ and 2c).

was formed by A growth front due to its fast growth rate. After the growth of B growth front, the compact-spherulite was formed with a characteristic of birefringence. It is expected that two growth fronts should be formed by two components respectively. Based on the spherulitic morphology and growth rate, the fast growth front in Figure 2 should be formed by PEO component. ${ }^{8}$

Obviously, the crystallization rate of PEO is much faster than that of PES. That means the crystallization of PES and PEO may not stop simultaneously though they nucleated simultaneously. The PES component may continue to crystallize after the PEO component crystallized completely and stopped to grow. Therefore, it was observed that the crystallization peaks in DSC curves were asymmetric even a shoulder or a small peak appeared at slower cooling rate for 20/ 80 and 30/70 samples at late crystallization stage as shown in Figure 1a and 1b. However, this phenomenon is difficult to be observed by means of polarizing optical microscope.

From Figure 2 PEO and PES always nucleate with the same primary nucleus to form blended spherulites. However it is found that PEO and PES can also nucleate alone at the cooling rate of $1.25^{\circ} \mathrm{C}$, as shown in Figure 3. Obviously, the nucleation mode of PEO and PES depends on whether PEO and PES crystallize simultaneously. Since the difference of crystallization temperature of PEO and PES decreases with increas-

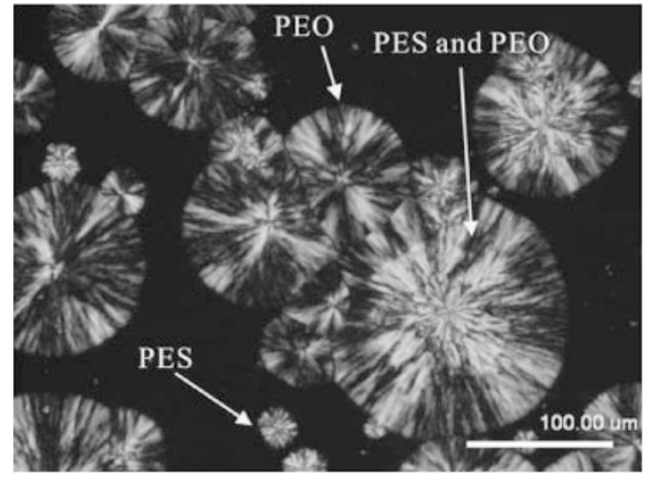

Figure 3. The spherulitic morphology of PES/PEO $=20 / 80$ blend with temperature at cooling rate of $1.25^{\circ} \mathrm{C}$ investigated by polarizing optical microscope. Three types of primary nuclei can be formed in this condition.

ing of cooling rate ( $c f$. Figure 1), PEO and PES can crystallize simultaneously under certain cooling rate for a certain composition of PES/PEO blend. In these cases, PEO and PES would nucleate with the same primary nucleus to form blended spherulites. The formation of blended spherulite is a unique crystallization behavior. According to the reference, ${ }^{8}$ PEO and PES can crystallize simultaneously with different nucleus under isothermal crystallization conditions. It can be considered that each growth front of PEO and PES from the same nucleus in the same spherulite maybe comes from the non-isothermal crystallization conditions, and the nucleation mode is different. How- 


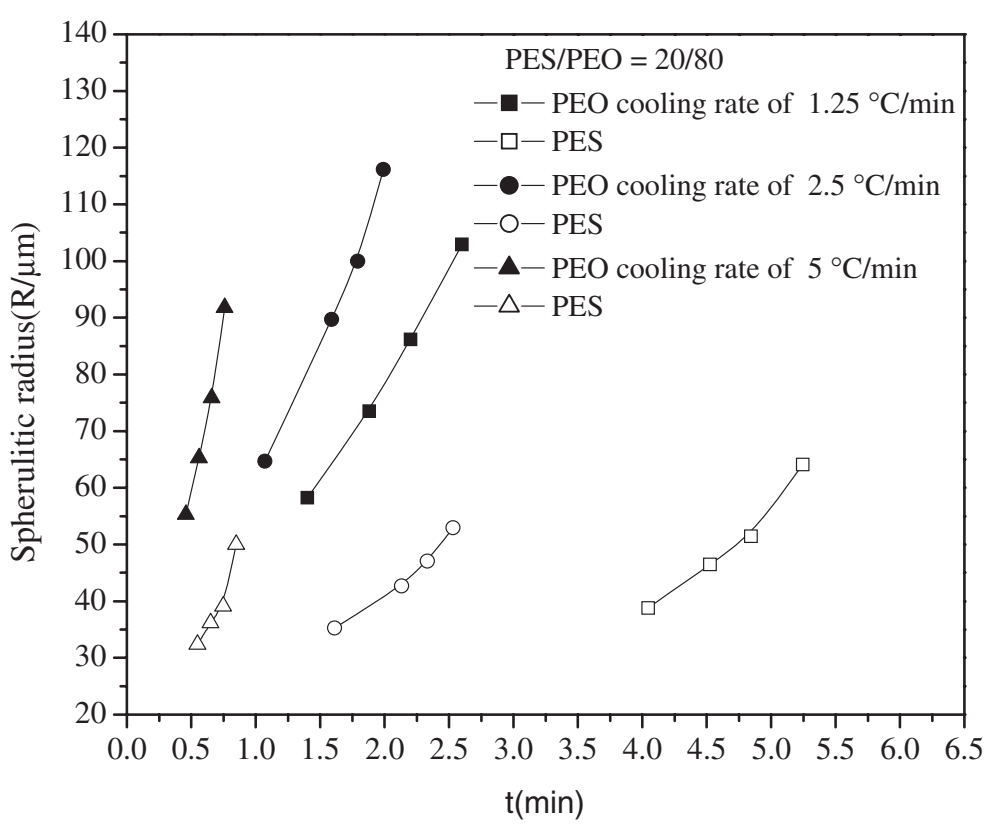

Figure 4. Radius of PES and PEO spherulites in a 20/80(wt/wt) PES/PEO blend with crystallization time at different cooling rates.

ever, this unique mechanism of spherulitic growth is not clear and need further study.

Figure 4 shows the increase of spherulitic radius of PEO and PES with crystallization time at different cooling rate. The growth rates of PEO and PES spherulites increased with crystallization time. Usually, the crystallization rate should increase with rising crystallization time under the non-isothermal crystallization condition due to the increase of supercoolings. According to references, ${ }^{16-19}$ when one component in a polymer blend crystallizes while the other component is still melting state, the melting component may be included in or rejected from the spherulites of the crystallized component. If the melting component is rejected from the spherulites of crystallized component, the growth rate of spherulites of crystallized component would decrease with time due to the concentration increase of the melting component at the growth front of crystallized component. Therefore, the result shown in Figure 4 indicated that non-crystalline PES melt should be included in the PEO spherulites when PEO crystallized firstly at higher temperature ( $c f$. Figure 2) and PES could then crystallize inside the PEO spherulites.

Figure 5 showed the crystallization and melting process of PES/PEO blend with $\mathrm{PEO} / \mathrm{PES}=60 / 40$ at cooling rate of $5{ }^{\circ} \mathrm{C}$. In this case, one component will start to crystallize after one component crystallized completely, which is different to the crystallization behavior of 20/80 sample. The final spherulite morphology does not show birefringence after both components crystallized completely. When the melting temperature is higher than the melting point of
PEO $\left(\sim 67^{\circ} \mathrm{C}\right)$, the morphology of spherulite changed and showed a characteristic of birefringence, which is the same as that before the second component crystallization. The melting experiment evidenced that PES crystallized at higher temperature than that of PEO in this condition.

From Figures $2 \mathrm{~d}$ and $5 \mathrm{~d}$, the final spherulitic morphologies formed by $20 / 80$ and $60 / 40$ samples at cooling rate of $5{ }^{\circ} \mathrm{C}$ are different after both components crystallized completely. As shown in Figure 2, the aggregated density of PEO lamellae is very small after PEO growth (A growth front) due to fast growth rate, and the open-spherulites of PEO was formed and there is no birefringence in PEO spherulites. The PES melt exists in interlamellae of PEO. When PES grew radically inside the PEO spherulite the lamellae of PES developed along with those of PEO. The lamellae of both components are interlocked each other, leading to the birefringence of spherulite. This type of growth of PES is similar to the interpenetrated growth reported by references ${ }^{6-10}$ while the growth direction is opposed, i.e., opposed-interpenetrated growth. For the step-crystallization of PES/PEO blend with composition of 60/40, the compact-spherulites of PES were first formed at higher temperature according to the formation of the spherulitic birefringence, and a great number of the melt phase of PEO should exist in the interfibrillar region of PES spherulites. Therefore the birefringence of spherulites resulted from the PES crystallization disappeared after PEO crystallized. The details of structural development will be discussed later by means of small angle X-ray scatter method. 


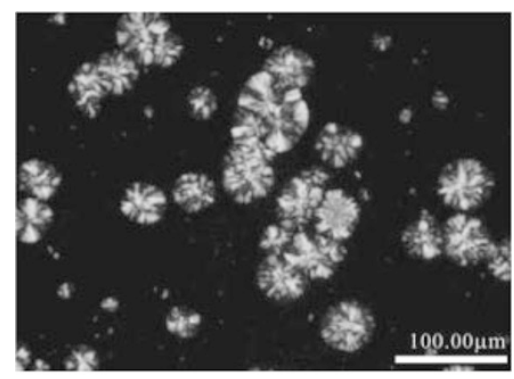

(a) $T=45.5^{\circ} \mathrm{C}$

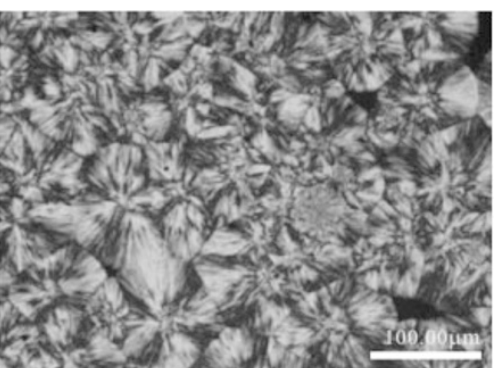

(c) $\mathrm{T}=44.3^{\circ} \mathrm{C}$

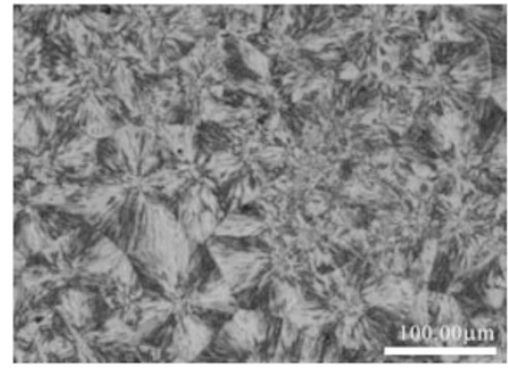

(e) $T=91.2^{\circ} \mathrm{C}$

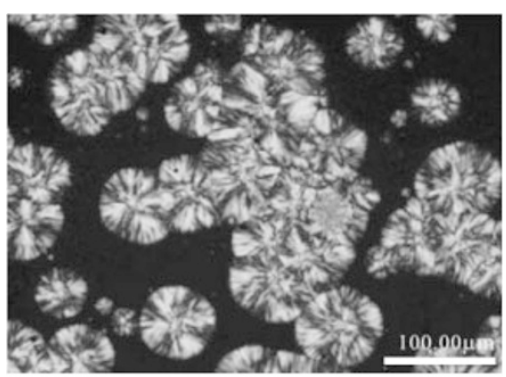

(b) $T=44.9^{\circ} \mathrm{C}$

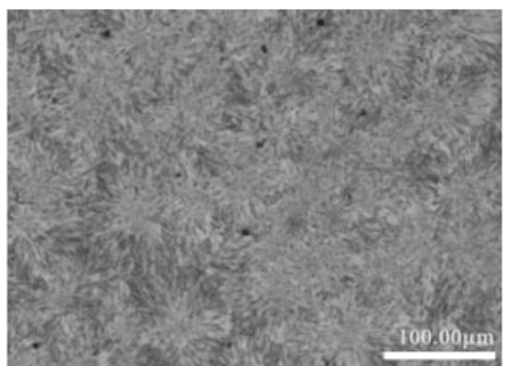

(d) $T=43.5^{\circ} \mathrm{C}$

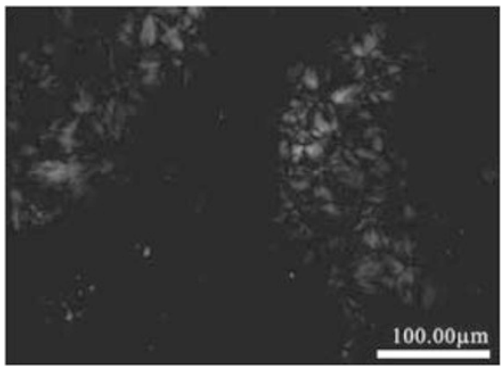

(f) $T=106.1^{\circ} \mathrm{C}$

Figure 5. The crystallization and melting process of PES/PEO blend with composition of $60 / 40$ at cooling rate of $5{ }^{\circ} \mathrm{C}$ with temperature of (a) $T=45.5^{\circ} \mathrm{C}$, (b) $T=44.9^{\circ} \mathrm{C}$, (c) $T=44.3{ }^{\circ} \mathrm{C}$, (d) $T=43.5^{\circ} \mathrm{C}$, (e) $T=91.2^{\circ} \mathrm{C}$, (f) $T=106.1{ }^{\circ} \mathrm{C}$ respectively. The change of spherulitic morphology can be observed clearly after the melting temperature is higher than melting point of PEO crystal.

\section{CONCLUSIONS}

Depending on the crystallization conditions and compositions of PES/PEO blends, PES and PEO can not only crystallize simultaneously but also crystallize upon step-crystallization. In the cases where PES and PEO crystallized simultaneously, a novel spherulitic growth was observed. PES and PEO nucleate with the same nucleus, and the growth fronts of PEO and PES components grew simultaneously with different growth rate in the same spherulite. PES spherulite grew radically inside the spherulite formed by PEO component due to the slower growth rate of PES, and the PES lamellae developed along the preexisting PEO lamellae to form an opposed-interpenetrated growth. In the cases where PES and PEO crystallized upon step crystallization, PES nucleated and grew firstly with the PEO melt phase in the interfibrillar regions. The morphology of spherulite changed after PEO crystallized. The results indicated that the final spherulitic morphology of a binary crystalline polymer blend should be controlled by the crystallization of the second crystallization component.

Acknowledgment. Financial supports by Science Foundation of Anhui and Key Lab Foundation of Anhui are gratefully acknowledged.

\section{REFERENCES}

1. H. D. Keith, F. J. Padden, and T. P. Rusell, Macromolecules, 22, 666 (1989).

2. Y. W. Cheung, R. S. Stein, J. S. Lin, and G. D. Wignall, Macromolecules, 27, 2520 (1994).

3. S. Talibuddin, L. Wu, J. Runt, and J. S. Lin, Macromolecules, 29, 7527 (1996).

4. J. P. Penning and R. St. John Manley, Macromolecules, 29, 77 (1996).

5. A. Dufresne and M. Vincendon, Macromolecules, 33, 2998 (2000).

6. J. C. Lee, H. Tazawa, T. Ikehara, and T. Nishi, Polym. J., 30, 780 (1998). 
7. T. Ikehara, Y. Nishikawa, and T. Nishi, Polymer, 44, 6657 (2003).

8. Z. Qiu, T. Ikehara, and T. Nishi, Macromolecules, 35, 8251 (2002).

9. T. Ikehara, H. Kimura, and Z. Qiu, Macromolecules, 38, 5104 (2005).

10. J. Liu and B. J. Jungnickel, J. Polym. Sci., Part B: Polym. Phys., 41, 873 (2003).

11. H. D. Keith and F. J. Padden, J. Appl. Phys., 34, 2409 (1963).

12. H. D. Keith and F. J. Padden, Polymer, 27, 1463 (1986).

13. B. J. Jungnickel, Res. Trends Curr. Trends Polym. Sci., 2,
157 (1997).

14. R. S. Stein, F. B. Khambatta, F. P. Warner, T. Russell, A. Escala, and E. Balizer, J. Polym. Sci., Polym. Symp., 63, 313 (1978).

15. H. L. Chen and S. F. Wang, Polymer, 41, 5157 (2000).

16. H. Tanaka and T. Nishi, Phys. Rev. Lett., 55, 1102 (1985).

17. E. Martuscelli, Polym. Eng. Sci., 24, 563 (1984).

18. H. Tanaka and T. Nishi, Phys. Rev. A: At., Mol., Opt. Phys., 39, 783 (1989).

19. R. Wellscheid, J. Wust, and B. J. Jungnickel, J. Polym. Sci., Part B: Polym. Phys., 34, 893 (1996). 